\title{
Análise comparativa das taxas de deterioração de sementes ortodoxas de Erythrina speciosa e recalcitrantes de Eugenia spp.
}

\author{
Isabela Pedroni Amorim' ${ }^{1}$, Joyce Generoso Souza' ${ }^{1}$ (1) \& Claudio José Barbedo ${ }^{1, *}$ (b) \\ 'Instituto de Botânica, Núcleo de Pesquisa em Sementes, Avenida Miguel Stéfano, 3687, 04301-012 São Paulo, SP, Brasil \\ * Autor para correspondência: cjbarbedo@yahoo.com.br
}

Recebido em 19.XII.2019

Aceito em 24.VI.2021

DOI 10.21826/2446-82312021v76e2021013

\begin{abstract}
RESUMO - Os principais fatores que afetam a capacidade de armazenamento de sementes são o teor de água e a temperatura do ambiente. Quanto mais altos esses valores, maiores as taxas de deterioração. Existem modelos matemáticos que propõem estimar a vida útil das sementes em armazenamento, uma vez que esses dois fatores são fornecidos. No entanto, para algumas espécies, especialmente em florestas nativas de regiões tropicais, essas equações não são tão eficientes. Neste trabalho, analisamos as taxas de deterioração de sementes tolerantes (Erythrina speciosa) e sensíveis (Eugenia spp.) à dessecação para termos mais elementos na busca do entendimento dessas diferenças na taxa de deterioração em diferentes condições ambientais. Os resultados mostraram grandes variações nas respostas, evidenciando a necessidade de mais estudos, principalmente quando as sementes são sensíveis à dessecação.
\end{abstract}

Palavras-chave: armazenamento de sementes, equações de viabilidade, sementes tropicais.

\begin{abstract}
A comparative analysis of deterioration rate between seeds of the orthodox Erythrina speciosa and recalcitrant Eugenia spp. species). The main factors affecting seed storage capacity are the seed water content and temperature. The higher are these values, the higher are the deterioration rates. There are mathematical models that allow to estimate the lifespan of seeds in storage, whenever the values of both factors are provided. However, for some species, especially these native from tropical forest regions, these equations are not so efficient. In this work we analysed the deterioration rates of desiccation-tolerant (Erythrina speciosa) and desiccation-sensitive (Eugenia spp.) seeds, in order to obtain new data to understand the difference in the deterioration rates under different environmental conditions. The results evidenced great variation in the responses. Therefore, more studies are needed, especially for seeds that are sensitive to desiccation.
\end{abstract}

Keywords: seed storability, tropical seeds, viability equations.

\section{INTRODUÇÃO}

O armazenamento de sementes em bancos de germoplasma é o método mais utilizado de conservação dos recursos fitogenéticos. Para isso, é necessário reconhecer as características fisiológicas das sementes de cada espécie, para só então selecionar as formas de processamento e de armazenamento adequadas e garantir a viabilidade prolongada e a preservação do material genético (Hong et al. 1996, Chaves \& Usberti 2003, Carvalho \& Nakagawa 2012). Além disso, algumas espécies possuem sementes que toleram a criopreservação e, dessa forma, podem ser armazenadas por um tempo ainda maior que nos métodos tradicionais, sendo mantidas sob temperatura ultrabaixa (nitrogênio líquido), com mínimo risco de perda do material biológico (Araújo et al. 2019).

Contudo, face à grande diversidade de espécies, especialmente nas regiões tropicais úmidas, verifica-se grande variação de comportamento das sementes, especialmente o relacionado à fisiologia da deterioração, dificultando o correto diagnóstico do potencial de armazenamento e a padronização de tecnologia para conservar as sementes de todas as espécies.

Condições adequadas de armazenamento, evidentemente, não podem aumentar a qualidade das sementes, mas permitem que sementes viáveis assim se mantenham por longos períodos quando se consegue reduzir a taxa de deterioração. As principais condições que interferem no armazenamento são a umidade relativa do ar, que frequentemente condiciona o teor de água das sementes, e a temperatura do ambiente (Vieira et al. 2001, Marcos-Filho 2015). Para um grande número de espécies, cujas sementes são tolerantes à redução do teor de água para valores inferiores a $10 \%$, genericamente denominadas ortodoxas, quanto maiores os valores dessas condições, maiores as taxas de deterioração e, portanto, menor o tempo de conservação da viabilidade. Contudo, há sementes, denominadas recalcitrantes, que não toleram redução do teor de água para valores inferiores a $12-15 \%$, considerados adequados ao armazenamento. A sensibilidade à dessecação não é uma 
situação de sim ou não (Berjak \& Pammenter 2008), mas varia entre espécies, produzindo categorias intermediárias (Dal Ri et al. 2017) e pode ocorrer até entre genótipos da mesma espécie (Lamarca et al. 2016, Lavanhole et al. 2019). Portanto, para as sementes não ortodoxas as informações são insuficientes para qualquer generalização de comportamento (Barbedo et al. 2013).

Para as sementes que toleram a redução no teor de água para valores inferiores a $10 \%$ há, na literatura, modelos matemáticos que se propõem a estimar o tempo de vida útil das sementes em armazenamento, como as equações de previsibilidade de armazenamento desenvolvidas por Roberts (1973) e Ellis \& Roberts (1980). Os modelos baseiam-se na relação entre o binômio temperatura - teor de água e a taxa de deterioração das sementes, estimandose que, dentro de determinados valores adequados ao armazenamento, quanto mais elevados temperatura e teor de água, mais rápida a taxa de deterioração (Marcos Filho 2015). A proximidade dos resultados experimentais com os previstos pelos modelos, principalmente quando utilizadas espécies agrícolas, permitiu a inclusão de tais fórmulas no manejo de lotes de sementes em bancos de germoplasma, como o do Millenium Seed Bank (Royal Botanic Garden 2019). Contudo, para algumas espécies, especialmente as florestais nativas de regiões tropicais, essas equações não se mostram tão eficientes (Santos \& Barbedo 2017).

Diversos fatores podem contribuir para as diferenças nos resultados, tais como as condições climáticas durante a formação das sementes, o beneficiamento, as condições e o período em que foram transportadas e armazenadas até que se realizaram os experimentos, o grau de maturação no momento da colheita, entre outros (Barbedo 2018, Hay et al. 2019). As condições nas quais os experimentos são realizados também podem interferir como, por exemplo, quando se utilizam condições experimentais muito distantes das reais, quando o universo amostral não é suficiente para um correto diagnóstico (Santos \& Barbedo 2017) e quando não há observação da dinâmica de movimentação de água, ou seja, se a semente está em absorção ou em dessorção (Hay et al. 2019). Contudo, mesmo se tomando o cuidado com essas variáveis, não há garantia de que um modelo possa ser universalizado para todas as espécies. Isso porque há grande variação nas características finais das sementes em cada ano e/ou local de produção em função das condições climáticas nas quais são desenvolvidas, inclusive para a mesma espécie e mesmo indivíduo (Barbedo 2018).

Para diversas espécies, embora não seja possível prever a vida útil de uma determinada semente, pode-se estimar o tempo de vida médio da população ou lote. Roberts (1973), por exemplo, desenvolveu modelos experimentais relacionando temperatura, teor de água e período médio de viabilidade de lote de sementes. Posteriormente, algumas mudanças sutis foram feitas nas equações, chegando a um novo modelo, que permitiu determinar coeficientes para cada espécie e levar em consideração a complexa dependência que a longevidade das sementes tem sobre a temperatura de armazenamento, bem como a importância da qualidade inicial do lote (Ellis \& Roberts 1980). No entanto, fatores que influenciam as constantes das equações são pouco estudados cientificamente e alguns estudos não têm obtido o sucesso esperado com a utilização desses modelos (Santos \& Barbedo 2017). Esse sucesso pode ser ainda menor quando se consideram as sementes recalcitrantes, que não são incluídas nos estudos por terem sementes sensíveis à dessecação.

Portanto, a ação da temperatura e do teor de água das sementes sobre as taxas de sua deterioração, embora amplamente estudada, ainda não está plenamente compreendida. Assim, o objetivo deste trabalho foi analisar as taxas de deterioração de sementes tolerantes e sensíveis à dessecação submetidas a diferentes condições de temperatura e umidade, a fim de compreender a diferença verificada no sucesso da aplicação daqueles modelos de previsibilidade de armazenamento de sementes.

\section{MATERIAL E MÉTODOS}

Utilizaram-se, como modelo experimental, quatro espécies nativas da flora brasileira, uma com sementes ortodoxas (Erythrina speciosa Andrews - Fabaceae, de duas regiões) e três com sementes reacalcitrantes (Eugenia brasiliensis Lam., E. involucrata DC. e E. uniflora L. - Myrtaceae).

Obtenção do material vegetal - As sementes de $E$. speciosa foram obtidas de matrizes do Jardim Botânico de São Paulo (2338'29" S, 46 37'24" O) e do Museu Catavento $\left(23^{\circ} 32^{\prime} 38^{\prime \prime} \mathrm{S}, 46^{\circ} 37^{\prime} 40^{\prime \prime} \mathrm{O}\right)$, coletadas em 2011 e armazenadas até 2014 (doravante denominadas E-2011/2014) e 2015 (doravante denominadas E-2011/2015) em sacos de papel, em temperatura ambiente $\left(22 \pm 7^{\circ} \mathrm{C}\right.$, $85 \pm 15 \%$ UR); as de E. brasiliensis, E. involucrata e E. uniflora, de matrizes do Jardim Botânico de São Paulo, em 2015 e 2016. As sementes foram extraídas dos frutos manualmente e, imediatamente antes do início dos experimentos, foram caracterizadas quanto ao teor de água, ao conteúdo de massa seca e à germinação, conforme descrito abaixo. Ao final dos períodos de incubação nas diferentes temperaturas, conforme descrito abaixo, as sementes foram novamente avaliadas quanto ao teor de água e à germinação.

Avaliações física e fisiológica das sementes - A determinação do teor de água e do conteúdo de massa seca foi realizada por secagem em estufa a $103 \pm 2^{\circ} \mathrm{C} /$ $17 \mathrm{~h}$ e os resultados foram calculados em base úmida (Brasil 2009). Por possuírem dormência física devido à impermeabilidade do tegumento, as sementes de $E$. speciosa foram escarificadas do lado oposto ao eixo embrionário, até que o cotilédone ficasse visível. Os testes de germinação foram conduzidos em câmara do tipo sala, climatizada a $25^{\circ} \mathrm{C}$, na presença de luz contínua. Para cada tratamento foram feitas 4 repetições com 20 sementes cada, acondicionadas em caixas de germinação (poliestireno transparente com $11 \times 11 \times 3,5 \mathrm{~cm}$, com tampa), preenchidas 
com três folhas de papel de germinação, umedecidas até sua saturação (cerca de 2,5 vezes o peso do papel, Brasil 2009), para os experimentos de temperaturas amenas, ou preenchidas até a metade com vermiculita de granulação média, umedecida até sua saturação, para os experimentos de temperaturas elevadas. As sementes foram colocadas sobre papel ou sobre vermiculita, tomando-se o cuidado, para E. speciosa, de manter o lado escarificado para baixo. Foram consideradas como germinadas as sementes que emitiram raiz de, no mínimo, $0,5 \mathrm{~cm}$.

Avaliação das taxas de deterioração de sementes tolerantes à dessecação, em temperaturas amenas Procurou-se avaliar as taxas de deterioração das sementes conforme o modelo proposto por Roberts (1973). Para tanto, sementes de E. speciosa de E-2011/2014 foram divididas em três grupos. No primeiro, foram mantidas com seu teor de água inicial (11\%); nos outros dois, foram submetidos a hidratação controlada, até que atingissem, respectivamente, $15 \%$ e $25 \%$ de água. Para isso, as sementes foram colocadas sobre redes de alumínio dentro das caixas de germinação com uma camada de água no fundo, de maneira que as sementes não entrassem em contato direto com a água. As sementes com cada grau de hidratação foram armazenadas em embalagens herméticas (frascos de vidro com tampa), dentro de câmaras incubadoras do tipo "Biochemical Oxygen Demand" (BOD), a 5, 15 e $25^{\circ} \mathrm{C}$, por 30 e 90 dias.

Avaliação das taxas de deterioração de sementes tolerantes à dessecação, em temperaturas elevadas Procurou-se avaliar as taxas de deterioração das sementes conforme o modelo proposto por Roberts \& Ellis (1989). Novamente, parte das sementes de E. speciosa, de E-2011/2015, foi mantida com seu teor de água inicial (10\%), enquanto outros dois foram submetidos à hidratação controlada até que atingissem, respectivamente, $17 \%$ e $29 \%$ de água. As sementes com cada grau de hidratação foram incubadas em embalagens herméticas, dentro de câmaras incubadoras do tipo BOD, a 40 e $50^{\circ} \mathrm{C}$, por 24 horas.

Avaliação das taxas de deterioração de sementes sensíveis à dessecação - Com base em resultados anteriores de tolerância à dessecação e a elevadas temperaturas (Delgado \& Barbedo 2007; Oliveira et al. 2011; Françoso \& Barbedo 2014, 2016), sementes de E. brasiliensis, E. involucrata e E. uniflora foram submetidas a sucessivas secagens em estufa, com ventilação de ar forçada, a $40^{\circ} \mathrm{C}$, a fim de obter três graus de hidratação para cada espécie. Para as sementes de E. involucrata, os teores de água foram $60 \%$ (inicial), $50 \%$ e $38 \%$; as de E. uniflora, $55 \%$ (inicial), $48 \%$ e $42 \%$; as de E. brasiliensis, 50\% (inicial), $45 \%$ e $41 \%$. As sementes de $E$. brasiliensis com cada grau de hidratação foram incubadas a $40^{\circ} \mathrm{C}$ e a $50^{\circ} \mathrm{C}$, por 6 e 12 horas, as sementes de E. involucrata e E. uniflora foram incubadas a $35,40,45$ e $50^{\circ} \mathrm{C}$, por 24 e 48 horas.

Delineamento experimental, análise estatística e obtenção das constantes de viabilidade - $\mathrm{O}$ delineamento experimental foi o inteiramente casualizado, utilizando-se esquema fatorial $2 \times 3$ (temperatura $\mathrm{x}$ teor de água), para Erythrina speciosa e Eugenia brasiliensis em temperaturas elevadas, $2 \times 3 \times 3$ (período de incubação $\times$ temperatura $\mathrm{x}$ teor de água), para Erythrina speciosa em temperaturas amenas, $2 \times 3 \times 4$ (período de incubação $\times$ teor de água $\mathrm{x}$ temperatura) para Eugenia involucrata e Eugenia uniflora em temperaturas amenas. Os dados obtidos foram submetidos à análise de variância (teste $\mathrm{F}$ ), ao nível de significância de 5\%, e as médias foram comparadas entre si pelo teste de Tukey, também ao nível de 5\% (Santana \& Ranal 2004).

\section{RESULTADOS E DISCUSSÃO}

As sementes de E. speciosa de E-2011/2014 estavam, inicialmente, com $11 \%$ de água e $100 \%$ de germinação. Houve interação significativa entre os três fatores, período de incubação, temperatura e teor de água. Após 30 dias de armazenamento, as sementes de quase todos os tratamentos continuaram com taxas de germinação muito próximas da inicial, exceto aquelas armazenadas com $25 \%$ de água a $25^{\circ} \mathrm{C}$, que sofreram uma redução significativa na capacidade germinativa (Tab. 1). Com 90 dias de armazenamento, as sementes com $11 \%$ e $15 \%$ de água continuaram com taxas de germinação elevadas, independente da temperatura de armazenamento, mas as com $25 \%$ de água tiveram uma expressiva redução em sua capacidade germinativa nas três temperaturas, inclusive perdendo completamente a capacidade germinativa a $25^{\circ} \mathrm{C}$ (Tab. 1). Estes resultados sugerem que o teor de água das sementes de E. speciosa tem efeito maior sobre sua deterioração do que a temperatura de armazenamento.

Tabela 1. Germinação (\%) de sementes de Erythrina speciosa (E-2011/2014) com 11\%, 15\% e 25\% de água, incubadas por 30 e 90 dias sob temperaturas de $5^{\circ} \mathrm{C}, 15^{\circ} \mathrm{C}$ e $25^{\circ} \mathrm{C}$.

\begin{tabular}{|c|c|c|c|c|c|c|}
\hline \multirow{2}{*}{ Teor de água (\%) } & \multicolumn{3}{|c|}{30 dias } & \multicolumn{3}{|c|}{90 dias } \\
\hline & $5^{\circ} \mathrm{C}$ & $15^{\circ} \mathrm{C}$ & $25^{\circ} \mathrm{C}$ & $5^{\circ} \mathrm{C}$ & $15^{\circ} \mathrm{C}$ & $25^{\circ} \mathrm{C}$ \\
\hline 11 & $98 \mathrm{aA} a^{*}$ & $95 \mathrm{aA} a$ & $96 \mathrm{aA} a$ & $98 \mathrm{aA} a$ & $98 \mathrm{aA} a$ & $96 \mathrm{aA} a$ \\
\hline 15 & $98 \mathrm{aA} a$ & $98 \mathrm{aA} a$ & $100 \mathrm{aA} a$ & $100 \mathrm{aA} a$ & $96 \mathrm{aA} a$ & $95 \mathrm{aA} a$ \\
\hline 25 & $95 \mathrm{aA} a$ & $95 \mathrm{aA} a$ & $13 \mathrm{bB} a$ & $33 \mathrm{bA} b$ & $6 \mathrm{bA} b$ & $0 \mathrm{bB} b$ \\
\hline
\end{tabular}

Coeficiente de variação: 8,68\% $(\mathrm{p}<0,00001)$

*Médias seguidas pela mesma letra (letras minúsculas nas colunas, maiúsculas em linhas e itálico para comparações de período) não diferem pelo teste de Tukey (5\%). 
As sementes de E. speciosa de E-2011/2015 estavam, inicialmente, com $10 \%$ de água e $85 \%$ de germinação. A incubação sob temperaturas elevadas confirmou a importância do teor de água na manutenção da viabilidade dessas sementes. Com 10\% de água, as sementes incubadas a $40^{\circ} \mathrm{C}$ e $50^{\circ} \mathrm{C}$ permaneceram com germinação elevada (Tab. 2). Quando incubadas com $17 \%$ e $29 \%$ de água apresentaram substancial redução na germinação, chegando a perder totalmente a capacidade germinativa quando incubadas a $50^{\circ} \mathrm{C}$ com $29 \%$ de água (Tab. 2).

Não houve diferença entre os períodos de incubação das sementes de E. brasiliensis (6 e 12 horas), tampouco este fator apresentou interação significativa com os demais. Houve interação significativa entre temperatura e teor de água e a elevação da temperatura de 40 para $50^{\circ} \mathrm{C}$ acelerou a deterioração das sementes em todos os níveis de hidratação (Tab. 3). Com a redução do teor de água, as sementes deterioraram-se mais rapidamente a $40^{\circ} \mathrm{C}$; curiosamente, porém, a $50^{\circ} \mathrm{C}$ a redução de $50 \%$ para $40 \%$ de água tornou as sementes mais resistentes a essa deterioração. Diferente do que ocorre com as sementes tolerantes à dessecação, à medida que o teor de água de sementes sensíveis é reduzido, diminui-se o número de sementes germináveis, a taxa de germinação e o vigor. Portanto, o comportamento das sementes observado sob temperatura de $40^{\circ} \mathrm{C}$ era esperado, mas não o das incubadas a $50^{\circ} \mathrm{C}$.

De forma similar, sementes de E. involucrata (para as quais houve interação entre os três fatores) submetidas à

Tabela 2. Germinação (\%) de sementes de Erythrina speciosa (E-2011/2015) com 10\%, 17\% e 29\% de água, incubadas por 24 horas sob temperaturas de 40 e $50^{\circ} \mathrm{C}$.

\begin{tabular}{ccc}
\hline \multirow{2}{*}{ Teor de água (\%) } & \multicolumn{2}{c}{ Temperatura $\left({ }^{\circ} \mathrm{C}\right)$} \\
\cline { 2 - 3 } & 40 & 50 \\
\hline 10 & $80 \mathrm{aA}^{*}$ & $69 \mathrm{aA}$ \\
17 & $30 \mathrm{bA}$ & $9 \mathrm{bB}$ \\
29 & $18 \mathrm{bA}$ & $0 \mathrm{cB}$ \\
\hline
\end{tabular}

Coeficiente de variação: $16,73 \%(\mathrm{p}<0,01156)$

*Médias seguidas pela mesma letra (minúsculas nas colunas, maiúsculas nas linhas) não diferem pelo teste de Tukey $(5 \%)$. redução do teor de água de $60 \%$ para $50 \%$ e $38 \%$ parecem ter resistido melhor ao aumento das temperaturas de incubação, apresentando maiores taxas de germinação (Tab. 4). Sementes com $60 \%$ de água, quando incubadas à $50^{\circ} \mathrm{C}$, perderam completamente sua capacidade germinativa já após 24 horas, enquanto que $15 \%$ e $22 \%$ das que foram secas, respectivamente, até $50 \%$ e $38 \%$ de água mantiveram a viabilidade mesmo após 48 horas de incubação (Tab. 4). A mesma tendência de redução na taxa de deterioração pela ligeira redução no teor de água foi verificada em sementes de $E$. uniflora (interação significativa entre os três fatores) que, com $55 \%$ de água, não toleraram $45^{\circ} \mathrm{C}$, mas mantiveram a viabilidade, nesta mesma temperatura, quando tiveram redução do teor de água para $48 \%$ e $42 \%$ (Tab. 5).

A água dentro das sementes exerce grande influência sobre os processos de deterioração (Marcos Filho 2015). A literatura descreve que, quanto menor o teor de água das sementes e menor a temperatura do ambiente, menor sua atividade fisiológica e menor a atividade fisiológica dos agentes deterioradores (Kramer \& Kozlowski 1972). Contudo, os resultados obtidos para as sementes sensíveis à dessecação, neste trabalho, não permitem tal generalização. Com a redução do teor de água, ora a deterioração se acelerou, ora reduziu. Portanto, a influência do teor de água no processo de deterioração, nessas sementes, parece ser mais complexa do que simplesmente uma relação entre atividade metabólica e taxa de deterioração.

Tabela 3. Germinação (\%) de sementes de Eugenia brasiliensis com $40 \%, 45 \%$ e $50 \%$ de água, incubadas sob temperaturas de $40^{\circ} \mathrm{C}$ e $50^{\circ} \mathrm{C}$.

\begin{tabular}{ccc}
\hline \multirow{2}{*}{ Teor de água (\%) } & \multicolumn{2}{c}{ Temperatura $\left({ }^{\circ} \mathrm{C}\right)$} \\
\cline { 2 - 3 } & 40 & 50 \\
\hline 40 & $84 \mathrm{bA}^{*}$ & $54 \mathrm{cB}$ \\
45 & $92 \mathrm{abA}$ & $82 \mathrm{aB}$ \\
50 & $95 \mathrm{aA}$ & $66 \mathrm{bB}$ \\
\hline
\end{tabular}

Coeficiente de variação: $10,84 \%(\mathrm{p}<0,00288)$

*Médias seguidas pela mesma letra (minúsculas nas colunas, maiúsculas nas linhas) não diferem pelo teste de Tukey (5\%).

Tabela 4. Germinação (\%) de sementes de Eugenia involucrata com 38\%, 50\% e 60\% de água, incubadas por 24 horas e 48 horas sob temperaturas de $35^{\circ} \mathrm{C}, 40^{\circ} \mathrm{C}, 45^{\circ} \mathrm{C}$ e $50^{\circ} \mathrm{C}$.

\begin{tabular}{|c|c|c|c|c|c|c|}
\hline \multirow{2}{*}{$\begin{array}{l}\text { Temperatura } \\
\left({ }^{\circ} \mathrm{C}\right)\end{array}$} & \multicolumn{3}{|c|}{24 horas } & \multicolumn{3}{|c|}{48 horas } \\
\hline & $38 \%$ & $50 \%$ & $60 \%$ & $38 \%$ & $50 \%$ & $60 \%$ \\
\hline 35 & $80 \mathrm{aA} a^{*}$ & $88 \mathrm{aA} a$ & $85 \mathrm{aA} a$ & $77 \mathrm{aB} a$ & $90 \mathrm{aAB} a$ & $95 \mathrm{aA} a$ \\
\hline 40 & $75 \mathrm{aB} a$ & $87 \mathrm{aAB} a$ & $93 \mathrm{aA} a$ & $78 \mathrm{aA} a$ & $93 \mathrm{aA} a$ & $88 \mathrm{aA} a$ \\
\hline 45 & $68 \mathrm{aB} a$ & $88 \mathrm{aA} a$ & $38 \mathrm{bCa}$ & $68 \mathrm{aB} a$ & $87 \mathrm{aA} a$ & $5 \mathrm{bCb}$ \\
\hline 50 & $78 \mathrm{aA} a$ & $65 \mathrm{bA} a$ & $0 \mathrm{cB} a$ & $22 \mathrm{bA} b$ & $15 \mathrm{bAB} b$ & $0 \mathrm{bB} a$ \\
\hline
\end{tabular}

Coeficiente de variação: $13,91 \%(\mathrm{p}<0,00001)$

* Médias seguidas pela mesma letra (letras minúsculas nas colunas, maiúsculas em linhas e itálico para comparações de período) não diferem pelo teste de Tukey (5\%). 
Tabela 5. Germinação (\%) de sementes de Eugenia uniflora com 42\%, 48\% e 55\% de água, incubadas por 24 horas e 48 horas sob temperaturas de $35^{\circ} \mathrm{C}, 40^{\circ} \mathrm{C}, 45^{\circ} \mathrm{C}$ e $50^{\circ} \mathrm{C}$.

\begin{tabular}{|c|c|c|c|c|c|c|}
\hline \multirow{2}{*}{$\begin{array}{c}\text { Temperatura } \\
\left({ }^{\circ} \mathrm{C}\right)\end{array}$} & \multicolumn{3}{|c|}{24 horas } & \multicolumn{3}{|c|}{48 horas } \\
\hline & $42 \%$ & $48 \%$ & $55 \%$ & $42 \%$ & $48 \%$ & $55 \%$ \\
\hline 35 & $97 \mathrm{aA} a^{*}$ & $97 \mathrm{aA} a$ & $93 \mathrm{aA} a$ & $98 \mathrm{aA} a$ & $95 \mathrm{aA} a$ & $97 \mathrm{aA} a$ \\
\hline 40 & $92 \mathrm{aA} a$ & $97 \mathrm{aA} a$ & $95 \mathrm{aA} a$ & $83 \mathrm{bA} a$ & $68 \mathrm{bB} b$ & $77 \mathrm{bAB} b$ \\
\hline 45 & $55 \mathrm{bB} a$ & $70 \mathrm{bA} a$ & $3 \mathrm{bCa}$ & $28 \mathrm{cA} b$ & $17 \mathrm{cA} b$ & $0 \mathrm{cB} a$ \\
\hline 50 & $20 \mathrm{cA} a$ & $0 \mathrm{cB} a$ & $0 \mathrm{bB} a$ & $5 \mathrm{dA} b$ & $0 \mathrm{dA} a$ & $0 \mathrm{cA} a$ \\
\hline
\end{tabular}

Coeficiente de variação: $13,51 \%(\mathrm{p}<0,00004)$

* Médias seguidas pela mesma letra (letras minúsculas nas colunas, maiúsculas em linhas e itálico para comparações de período) não diferem pelo teste de Tukey $(5 \%)$.

A temperatura, por sua vez, parece apresentar respostas mais uniformes: quanto mais elevada, maior a taxa de deterioração. Portanto, nas equações de previsibilidade de conservação da viabilidade em armazenamento, a temperatura continuaria a ser um fator realmente previsível, com coeficiente sempre negativo (inversamente proporcional ao período de conservação). Por outro lado, o teor de água das sementes, para as sensíveis à dessecação, pelos dados aqui obtidos parece não apresentar sequer um padrão que permita relacioná-lo direta (coeficiente positivo) ou inversamente (coeficiente negativo) à conservação das sementes, não permitindo a construção das curvas de sobrevivência (em probits) e, consequentemente, não permitindo a obtenção de equações de previsibilidade.

Se, por um lado, o comportamento não padronizável das sementes sensíveis à dessecação (ao menos segundo os padrões esperados atuais) não permite incluir tais sementes em equações de previsibilidade, por outro lado a gigantesca margem de erro observada para as sementes tolerantes à dessecação deste trabalho, quando se modificam os modelos aplicados e as equações obtidas, também não permitiria obter previsões confiáveis do potencial de armazenamento dessas sementes. Deve-se salientar que o estudo foi realizado com espécie nativa do Brasil, não submetida a melhoramento genético e, portanto, com ampla variabilidade genética dentro de um mesmo lote. Contudo, os modelos atuais, aplicados às sementes de espécies agrícolas, tem se mostrado bastante eficientes nessa previsibilidade. A grande diferença entre essas respostas pode residir na diversidade genética, ampla naquelas e muito restrita nestas. De qualquer forma, os resultados apresentados neste trabalho mostram que é necessária muita cautela na aplicação das equações de previsibilidade, especialmente quando se trata de espécie nativa não domesticada e não submetida a processo de melhoramento vegetal. Além disso, ficou demonstrada a necessidade de maiores estudos quanto à influência do grau de hidratação das sementes sobre sua taxa de deterioração, principalmente quando as sementes são sensíveis à dessecação.

\section{AGRADECIMENTOS}

Os autores agradecem ao Conselho Nacional de Desenvolvimento Científico e Tecnológico - CNPq pela bolsa de iniciação científica (PIBIC) concedida a I.P. Amorim e J.G. Souza, ao Instituto de Botânica, pela permissão para as coletas e à Fundação de Amparo à Pesquisa do Estado de São Paulo (FAPESP), pelo apoio financeiro ao projeto 2017/50341-0.

\section{REFERÊNCIAS}

Araújo, D.S., Soares, F.S., Sobrinho, S.P. \& Luz, P.B. 2019. Crioprotetores na criopreservação de sementes de Passiflora mucronata Lam. Iheringia. Série Botânica 74: e2019008. Disponível em: https://isb. emnuvens.com.br/iheringia/article/view/543/483

Barbedo, C.J. 2018. A new approach towards the so-called recalcitrant seeds. Journal of Seed Science 40: 221-236.

Barbedo, C.J. \& Bilia, D.A.C. 1997. Germinação de sementes e desenvolvimento de plântulas de Inga uruguensis Hook. \& Arn. em função da remoção da sarcotesta. Informativo Abrates 7: 54-56.

Barbedo, C.J., Centeno, D.C. \& Figueiredo-Ribeiro, R.C.L. 2013. Do recalcitrant seeds really exist? Hoehnea 40: 583-593.

Berjak, P. \& Pammenter, N.W. 2008. Recalcitrance is not an all-or-nothing situation. Seed Science Research 4: 263-264.

Brasil. 2009. Ministério da agricultura, Pecuária e Abastecimento. Regras para análise de sementes. Secretaria da Defesa Agropecuária/MAPA, Brasília.

Carvalho, N.M. \& Nakagawa, J. 2012. Sementes: ciência, tecnologia e produção. 5 ed. Funep, Jaboticabal. 590 p.

Chaves, M.M.F. \& Usberti, R. 2003. Previsão da longevidade de sementes de faveiro (Dimorphandra mollis Benth.). Revista Brasileira de Botânica 26: 557-564.

Dal Ri, L., Calil, A.C., Silva, L.C. \& Müller, D.R. 2017. Comportamento da qualidade fisiológica de sementes de capororoca-do-banhado (Myrsine parviflora A. DC. - Primulaceae) em diferentes tempos e condições de armazenamento. Iheringia. Série Botânica 72: 403-408.

Delgado, L.F. \& Barbedo, C.J. 2007. Tolerância à dessecação de sementes de espécies de Eugenia. Pesquisa Agropecuária Brasileira 42: 265272.

Ellis, R.H. \& Roberts, E.H. 1980. Improved equations for the prediction of seed longevity. Annals of Botany 45: 13-30.

Françoso, C.F. \& Barbedo, C.J. 2014. Tratamentos osmóticos e térmicos para controle de fungos em sementes de grumixameira (Eugenia brasiliensis Lam.) e pitangueira (Eugenia uniflora L.). Hoehnea 41: 541-552. 
Françoso, C.F. \& Barbedo, C.J. 2016. Osmotic and heat treatments on control of fungi associated with seeds of Eugenia brasiliensis and E. pyriformis (Myrtaceae). Journal of Seed Science 38: 195-203.

Hay, F.R., Valdez, R., Lee, J.S. \& Cruz, P.C.S. 2019. Seed longevity phenotyping: recommendations on research methodology. Journal of Experimental Botany 70: 425-434.

Hong, T.D., Linington, S. \& Ellis, R.H. 1996. Seed storage behavior: A compendium. International Plant Genetic Resources Institute, Roma. 115 p.

Kramer, P.J. \& Kozlowski, T. 1972. Fisiologia das árvores. Fundação Calouste Gulbenkian, Lisboa. 745 p.

Lamarca, E.V., Camargo, M.B.P., Teixeira, S.P., Silva, E.A.A., Faria, J.M.R. \& Barbedo, C.J. 2016. Variations in desiccation tolerance in seeds of Eugenia pyriformis: dispersal at different stages of maturation. Revista Ciência Agronômica 47: 118-126.

Lavanhole, D.F., Arantes, S.D., Arantes, L.O., Souza, C.A.S., Posse, S.C.P., Cerri Neto, B. \& Crasque, J. 2019. Ecofisiologia da germinação dos genótipos de Theobroma cacao L. Iheringia. Série Botânica 74: e2019007. Disponível em: https://doi.org/10.21826/2446$82312019 \mathrm{v} 74 \mathrm{e} 2019007$

Marcos-Filho, J. 2005. Fisiologia de sementes de plantas cultivadas. Fundação de Estudos Agrários, Piracicaba. 495 p.
Marcos-Filho, J. 2015. Fisiologia de sementes de plantas cultivadas. 2 ed. ABRATES, Londrina. 659 p.

Oliveira, C.F., Oliveira, D.C., Parisi, J.J.D. \& Barbedo, C.J. 2011. Deterioração de sementes de espécies brasileiras de Eugenia em função da incidência e do controle de fungos. Revista Brasileira de Sementes 33: 520-532.

Roberts, E.H. 1973. Predicting the storage life of seeds. Seed Science and Technology 1: 39-52.

Roberts, E.H. \& Ellis, R.H. 1989. Water and seed survival. Annals of Botany 63: 39-52.

Royal Botanic Gardens Kew. 2019. Seed Information Database (SID). Disponível em http://data.kew.org/sid/viability/longevity.jsp (acesso em 03-06-2019).

Santana, D.G. \& Ranal, M.A. 2004. Análise da germinação: um enfoque estatístico. Editora UnB, Brasília. 248 p.

Santos, M.R.O. \& Barbedo, C.J. 2017. Deterioration rates of brazilwood seeds (Caesalpinia echinata Lam.) under high temperatures. Hoehnea 44: 449-463.

Vieira, A.H., Martins, E.P., Pequeno, P.L.L., Locatelli, M. \& Souza, M.G. 2001. Técnicas de produção de sementes florestais. EMBRAPACPAF, Rondônia. 4 p. 